\title{
Research on Vibration Risk Predictive Control of Auxiliary Shaft Hoist
}

\section{based on MCGS}

\author{
Yunkai Liu ${ }^{1, a}$, Jie Li ${ }^{2, b}$, Xiangzhong Meng ${ }^{3, c^{*}}$ and Tao Yin ${ }^{4, d}$ \\ ${ }^{1}$ Geting Coal Mine , Shandong energy Zibo mining group Co.,LTD.Jining, China \\ ${ }^{2}$ Geting Coal Mine, Shandong energy Zibo mining group Co.,LTD.Jining, China \\ ${ }^{3}$ College of Automation and Electronic Engineering, Qingdao University of Science and Technology, \\ Qingdao, China \\ ${ }^{4}$ College of Automation and Electronic Engineering, Qingdao University of Science and Technology, \\ Qingdao, China \\ cxzmengkd@163.com, d15275299920@163.com
}

Keywords: auxiliary shaft hoist; predictive control; vibration signal;MCGS

Abstract. For the existing problems of security risks when the amplitude of auxiliary shaft hoist vibration is too large, we use the vibration sensors to collect the vibration signals of auxiliary shaft hoist and display it in the monitoring software MCGS, then research on the vibration risk predictive control according to the analysis of the collecting signals integrated into signal processing, automatic control technology, and using PLC to control the converter so as to adjust the running state of the hoist., finally realizing the online monitoring of the hoist running status and vibration risk predictive control of auxiliary shaft hoist.

\section{Introduction}

Auxiliary shaft hoist is the main transmission device for auxiliary materials, equipment and staff of the coal mine, its security requirement is even higher than the main shaft hoist, its running state is normal or not will directly affect the quality and safety of coal mine production. The auxiliary shaft hoist always produce vibrations in each stage of the starting, acceleration, deceleration and constant speed. The overrun vibration and irregular vibration will accelerate the wear of the gears and other parts, which will shorten the service life of the hoist, and excessive vibration will decrease dynamic stiffness of the bearing, the bearing vibration intensified, which will increase the risk of failure ${ }^{[1]}$. In this paper, we use the PLC to send the vibration signals of the auxiliary shaft hoist collected by vibration sensors to the PC configuration software MCGS, MCGS exchanges data with MATLAB through the DDE protocol, and design the forecasting predictive controller in MATLAB to analyze the vibration signals and the control data is returned the lower computer MCGS, the PLC control inverter according to the instructions from MCGS so as to adjust the running status the auxiliary shaft hoist.

\section{The predictive control principle based on vibration signal analysis}

The vibration of the hoist is defined as the changing trend of the relative vibration displacement of bearing during running process, taking the vibration displacement as the basis of the monitoring and risk predictive control for the running status of the hoist. This system evaluates the size of control 
force required according to the external excitation and the amplitude of vibration displacement, and by adjusting the parameters of controller, finally realizing the soft-starting of the converter so as to adjust the running state of the hoist, which can effectively reduce the vibration response of the hoist ${ }^{[2]}$. Its predictive control works as follows: using the PLC to send the vibration signals of the auxiliary shaft hoist collected by vibration sensors to the PC configuration software MCGS, MCGS exchanges data with MATLAB through the DDE protocol, design the forecasting predictive controller in MATLAB to analysis the vibration signals and the control data is returned the lower computer MCGS , the PLC control inverter according to the instructions from MCGS so as to adjust the running status the auxiliary shaft hoist, finally enhance the stability and safety of the hoist' running state. The concrete structure of predictive control is shown in fig.1:

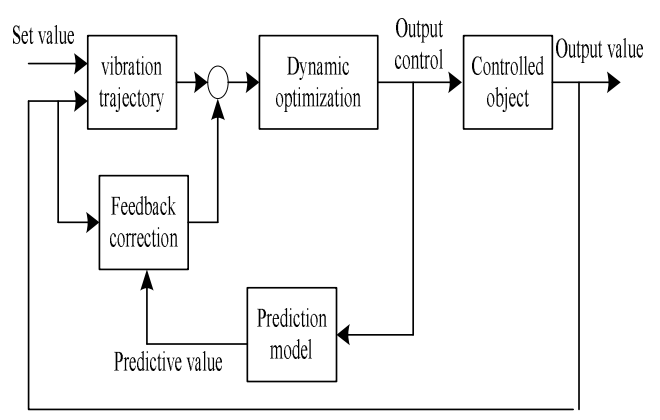

Fig.1. The structure diagram of predictive control

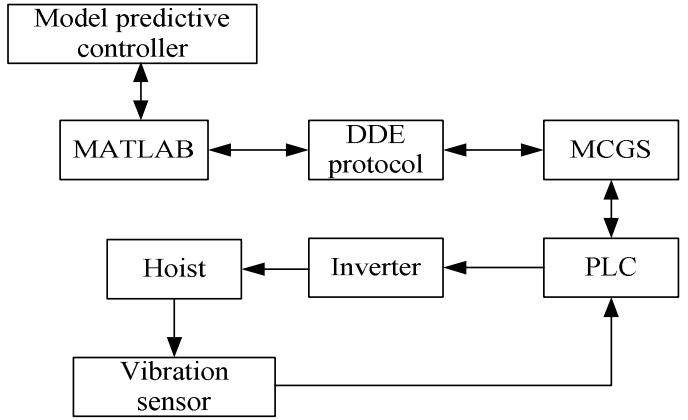

Fig.2. the control structure chart of system

The predictive model can predict the output according to the historical data of the vibration signal and the possibilities of the future input ${ }^{[3]}$. The feedback correction is mainly based on the actual output detected to modify the predictive error of the model, and taking the next step optimization. Dynamic optimization is based on the change in running status of the hoist and repeated optimization, it's the fundamental points which is different from the traditional predictive control optimal control. In addition, predictive control adopts multiple steps optimization ${ }^{[4]}$, compared with the traditional way of one step optimization, it can obtain more dynamic parameters that can reflect the running state trend of the hoist, thus it can accurately overcome the effects of various interference so that predictive control can get good results in the application of vibration risk predictive control of the hoist $^{[6]}$.

\section{The design of the system predictive controller}

The system uses the S-function block in the MATLAB/Simulink, namely model predictive controller module nlmpcsim, and take the simulation analysis of vibration characteristics of the hoist based on the running parameters collected by vibration sensors ${ }^{[5]}$. According to the results, using the predictive control algorithm to adjust the parameters of model online, then using the PLC to control the inverter to adjust the running status of the auxiliary shaft hoist according to the instructions from MCGS, to achieve the vibration risk predictive control of the hoist. The structure diagram of the system controlling as fig.2:

DDE protocol (Dynamic Data Exchange protocol) is a communication protocol that can exchange data between different software programs built-in MCGS configuration software, MATLAB also supports DDE protocol. In the vibration risk predictive control system of the auxiliary shaft hoist, the configuration software MCGS is the monitoring software, MAT LAB is applied as predictive control program in host computer. The monitoring vibration signal of the hoist is sent to the MATLAB from MCGS based on DDE protocol, and using the predictive controller to calculate the control force optimized, then return the control commands to the MCGS, using the PLC to achieve the automatic adjustment control of the hoist running state. 
In order to realize the DDE communication between MCGS and MATLAB, firstly define the variable in the "real-time database" of MCGS, and select "DDE connection management" in the configuration environment toolbar, then set the hoist data required as the DDE output in the basic settings.

MATLAB is the data processing program in host computer that supports various forms of data transfer, it can communicate with MCGS using the corresponding function provided by DDE module. MATLAB provides six DDE function, namely ddeinit, ddeterm, ddereq, ddepoke, ddeadv, ddeunadv ${ }^{[7]}$. Writing programs with this six function to send the MCGS data in the MATLAB, the calculation results will be returned to the main program.

The specific flow chart of predictive control is as follows:

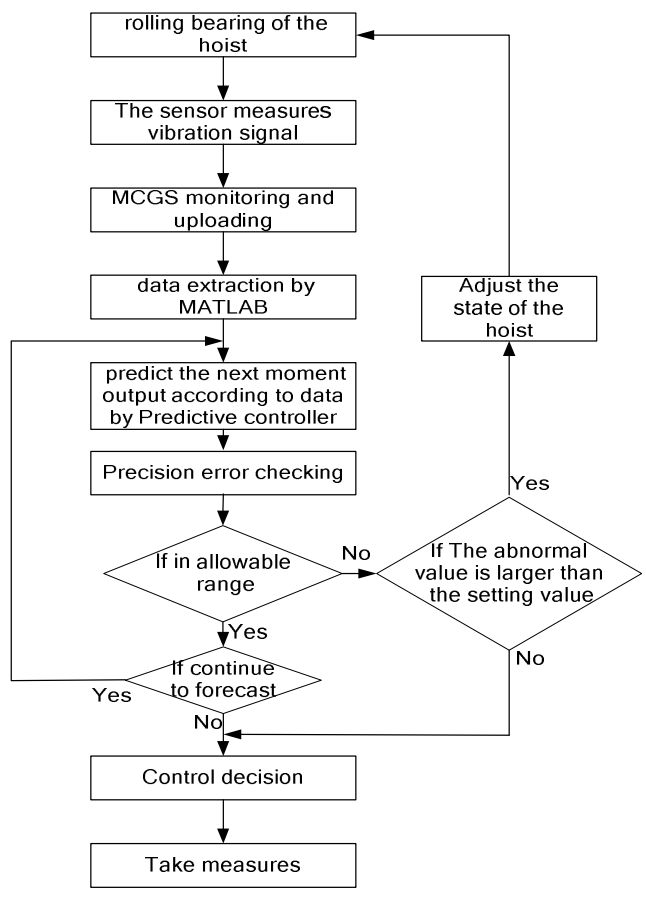

Fig.3. the flow chart of the vibration risk predictive control of the hoist

\section{Simulation analysis of predictive control for the hoist model}

We establish the vibration predictive control simulation model based on Matlab according to the dynamic characteristic parameters and the mathematical model of auxiliary hoist. and obtain the displacement waveform of the vibration signal through simulation while the hoist without predictive control and under predictive control, we also get the input and output information under the predictive control and analyze the control effect.

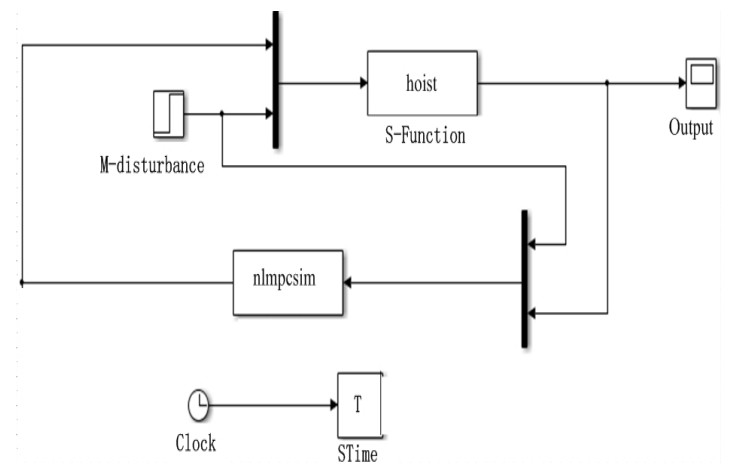

Fig.4. the simulation model of vibration risk predictive control for the hoist

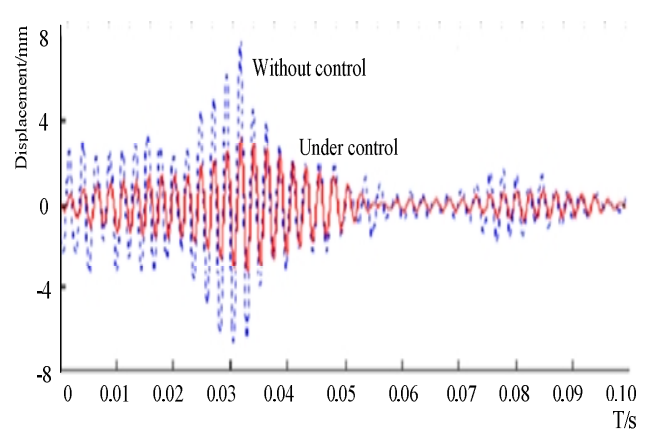

Fig.5. the displacement effect chart of predictive control for vibration signals in vertical direction

In Fig.4, the hoist module is the mathematical model of the hoist packaged by S-function, the 
nlmpcsim is predictive controller model, whose input consists of a vibration signal and part of interference that can be measured. and set the model parameter of predictive control toolbox in the MATLAB according to the main technical parameters of the hoist, for the convenience of calculation, the quality of reducer and head sheave in the model are set as $100 \mathrm{~kg}$, the stiffness coefficient is $k_{1}=k_{2}=k_{3}=980 \mathrm{kN} / \mathrm{m}$, and the acceleration of gravity is $10 \mathrm{~m} / \mathrm{s}^{2}$, set the simulation time as $0.1 \mathrm{~s}$, finally get the vibration signal simulation effect diagram in the vertical direction of the hoist from start to accelerate and uniform as fig.5.

From the displacement effect chart of predictive control for vibration signals in vertical direction, comparing with non predictive control, we can see that the control effect is better under the action of predictive control, the vibration amplitude decreases relatively with obvious damping effect, which can increase the stability and safety of the hoist running, it proves that the control system is feasible and effective, which has good application effect.

\section{Conclusions}

In this paper, according to the analysis of vibration signals of the auxiliary shaft hoist, we use PLC to realize data collection in the field with the MCGS configuration software, using the powerful data processing ability of Matlab, with the application of predictive control algorithm for vibration control of risk prediction, it can automatically adjust the running status of the auxiliary shaft hoist, reduce the risk of the hoist timely and effectively and increasing the stability and security of the hoist, which can provide guarantee for the normal production of the coal mine.

\section{References}

[1] Golay MW, et al. An integrated method for comprehensive sensor network development in complex power plant systems. Reliability Engineering and System Safety, 2000,(67):17-27.

[2] M.F.While.RollingElementBearingVibrationTransferCharaeteristies:Effect of Stiffness.Journal of Applied Mechanics.1979.Vol.46. 677 684.

[3] P.D.McFadden\&M.M.T00ZHY,Application of Synchronous Averaging to Vibration Monitoring of Rolling Bearings. Mechanical Systems and Signal Processing (2000)14(6),891 906.

[4] Kaewkongka T.,Joe Au Y.H., Rakowski R.et al. Continuous wavelets transform and neural network for condition monitoring of rotor-dynamic machinery. IEEE Instrumentation and Measurement Technology Conference.2001:1962 - 1966.

[5] D. Laurí, J. Sanchis, M. Martínez, A. Hilario. Latent variable based model predictive control: Ensuring validity of predictive .Journal of Process Control, 2013

[6] DING Bao-chang. The theory and method of predictive control[M].China Machine Press,2008

[7] LIANG Li. Control system simulation Based on the configuration software MCGS and MATLAB[J]. Industrial control computer,2005 